\title{
Comparison of amounts and types of practice during rehabilitation for traumatic brain injury and stroke
}

\author{
Teresa Jacobson Kimberley, PhD, PT; ${ }^{1-2 *}$ Sharyl Samargia, MA, CCC-SLP; ${ }^{2}$ Lisa G. Moore, DPT; $^{1}$ Josefin K. $^{2}$ \\ Shakya, DPT; ${ }^{1}$ Catherine E. Lang, PhD, PT $^{3}$ \\ ${ }^{1}$ Program in Physical Therapy, Department of Physical Medicine and Rehabilitation, and ${ }^{2}$ Program in Rehabilitation \\ Science, University of Minnesota, Minneapolis, MN; ${ }^{3}$ Program in Physical Therapy, Program in Occupational Therapy, \\ Department of Neurology, Washington University, St. Louis, MO
}

\begin{abstract}
Patients with acquired neurological deficits may capitalize on cortical reorganization to recover functional skills that have been lost. Research in neuroplasticity proposes that a high number of repetitions may lead to cortical reorganization. The purposes of this study were to quantify the number and type of activities performed by patients with traumatic brain injury (TBI) and stroke in physical and occupational therapy sessions to determine whether (1) the number of repetitions approaches the numbers in neuroplasticity research, (2) there were differences based on patient diagnosis, and (3) patient or therapist characteristics affected the type or amount of activities performed. Forty-eight patient and forty provider subjects participated. One hundred seven therapy sessions were observed. Data from therapy sessions were counted and categorized. Neither patient group approached the total number of repetitions neuroplasticity research suggests may be required for neuroplastic change. Repetitions per session did not differ between groups. Subjects with TBI performed more repetitions per minute in three categories (total upper-limb repetitions, gait steps, and transfers) than subjects with stroke. Therapists with $<1$ year or $>15$ years of neurological therapy experience instructed patients in fewer functional repetitions per minute than did therapists with 5 to 15 years of experience.
\end{abstract}

Key words: cortical reorganization, CVA, neuroplasticity, observation, occupational therapy, physical therapy, rehabilitation, repetitions, stroke, TBI.

\section{INTRODUCTION}

The process by which neuronal circuits are modified by experience, learning, or injury is referred to as neuroplasticity [1]. Knowledge of this process has greatly expanded in recent years, with important implications for rehabilitation. Both the brain and body need to relearn how to function following neurological injury, and harnessing this inherent ability for neuronal circuit change in the brain may be essential to maximize the benefit of rehabilitation. Motor-learning research in nondisabled subjects and subjects with neurological compromise has suggested that high numbers of repetitions (reps) of task-specific activity may be required to promote neuroplastic change. Animal studies in neuroplasticity have shown that 400 to 600 reps per day of a challenging functional task (fine-motor grasping) can lead to structural neurological changes following an induced stroke to the hand area in nonhuman primates

\footnotetext{
Abbreviations: CIMT = constraint-induced movement therapy, FIM = Functional Independence Measure, LL = lower limb, reps $=$ repetitions, $\mathrm{SD}=$ standard deviation, $\mathrm{TBI}=$ traumatic brain injury, UL = upper limb.

*Address all correspondence to Teresa Jacobson Kimberley, PhD, PT; University of Minnesota, MMC 388, 420 Church St SE, Minneapolis, MN 55455; 612-626-4096.

Email: tik@umn.edu

DOI:10.1682/JRRD.2010.02.0019
} 
[2-4]. In nondisabled humans, repeated practice of specific thumb movements can alter the transcranial magnetic stimulation evoked responses toward the trained direction [5]. This change required 15 to 30 minutes of continuous effort of one movement and extinguished after approximately 20 to 30 minutes. After stroke, increased amounts of task repetition have been shown to cause cortical changes and functional improvement [6-8]. As a specific example, in Carey et al., subjects with impaired grasp-and-release secondary to stroke performed more than 100 reps per day (1,200 total) of a finger-tracking exercise and demonstrated significant cortical reorganization and functional improvement compared with control subjects [6]. In the lower limb (LL), gait evidence in animal models suggests that approximately 1,000 to 2,000 steps per session are required to improve hind-limb stepping and step quality [9-11].

While task repetition is not the only important feature, it is becoming clear that neuroplastic change and functional improvement occur after large numbers of a specific task are performed but do not occur with fewer numbers [12-13]. Thus, one item of focus for rehabilitation professionals should be the number of reps and type of activity performed. However, very little research is available that quantifies the amount and type of movement practice that occurs during a clinical rehabilitation session [14-16]. In a pilot study [16] and a larger multicenter study across North America [17], practice of taskspecific functional upper-limb (UL) movement occurred in only 51 percent of sessions that addressed the UL and the average number of reps per session was 32 . The average number of gait steps performed per session was 357 . These findings are an order of magnitude lower than the number of reps documented in neuroplasticity research.

As with stroke, patients with traumatic brain injury (TBI) also require cortical reorganization to overcome neurological damage. Occupational and physical therapy settings are experiencing increasing numbers of patients with TBI on their caseloads because of advances in medical technology that allow more people to survive brain injury. According to Rutland-Brown et al., of the approximately 1.57 million Americans who sustained TBI in 2003, 97 percent survived [18]. TBI is also noted as the "signature injury" of Operation Iraqi Freedom and Operation Enduring Freedom. More than 12,000 members of the armed forces reported TBI from March 2003 to March 2007 [19], thereby increasing the prevalence of TBI in the United States and the number of patients requiring rehabilitation.
Limited research describes and quantifies the rehabilitation of patients with this diagnosis.

Patients with TBI have been shown to have positive rehabilitation outcomes as a result of high numbers of functional reps, such as is required in constraint-induced movement therapy (CIMT) [20]. Patients with TBI who received additional functional reps (+160 reps of sit-tostand and step-ups) had a larger functional improvement compared with a group who did not receive the additional reps, suggesting that the dose of rehabilitation is also important in TBI [21]. No studies to date have documented the number of reps typically performed by patients with TBI during a clinical therapy session. Similarly, the extent to which patient diagnosis or therapist characteristics affect number of reps performed by patients is unknown.

The purposes of this study were to quantify the number and type of activities performed by patients with TBI and stroke in physical and occupational therapy sessions to determine whether (1) the number of reps approaches the numbers in neuroplasticity research, (2) there were differences based on patient diagnosis, and (3) patient or therapist characteristics affected the type or amount of activities. This comparison between diagnoses allows insight into whether this limited repetition number is unique to stroke or whether it occurs in another major diagnostic category as well.

\section{METHODS}

\section{Subjects}

This observational study was conducted at two metropolitan-area hospital settings: an acute hospital and a long-term acute rehabilitation facility. Both the rehabilitation providers and patients were considered subjects and both diagnoses were seen at each facility. The rehabilitation providers were referred to as "provider subjects" and the patients were referred to as "patient subjects." Provider subjects were included in the study if they were a licensed physical therapist $(n=17)$, physical therapist assistant $(n=$ 7 ), occupational therapist ( $n=13$ ), or certified occupational therapist assistant $(n=3)$. A total of 50 provider subjects consented, 40 of whom (3 male, 37 female) were observed. Provider subjects were divided into five groups based on their years of neurorehabilitation experience: $<1$ year $(n=3), 1$ to $<5$ years $(n=13), 5$ to $<10$ years $(n=7), 10$ to $<15$ years $(n=6)$, and $>15$ years $(n=11)$. 
A total of 48 patient subjects (29 male, 19 female) participated in the study. All patient subjects were referred for physical or occupational therapy. Patients with unilateral or bilateral paresis due to stroke or TBI met inclusion criteria. Patient subjects were excluded if they or a family member could not provide informed consent or if the functional level of the patient prevented any active limb movement. Patient subjects were then divided into two groups based on diagnosis. There were 24 patient subjects in the TBI group and 24 patient subjects in the stroke group.

The stroke group in this study was part of a larger multicenter study, and observers were trained to correctly classify each therapy exercise through written materials and videos that were developed for use in the pilot study by Lang et al. [16]. Each observer was then tested for reliability, requiring a score of at least 90 percent in order to collect study data. Previous use of this method resulted in an interrater reliability intraclass correlation coefficient of $0.99(p<0.001)$. The observer obtained the data by recording each repetition within a therapy session and placing the count in its designated category. Observers positioned themselves so they did not interfere with the therapy session yet could still hear and see what took place. Observers had no direct contact with the subjects during the treatment sessions but could approach the therapist after the session in order to clarify the purpose of an activity to ensure correct classification and documentation. The same observation procedure was used during all observations, regardless of patient subject diagnosis.

Three trained observers conducted a total of 107 observations (53 stroke, 54 TBI; range, mean age \pm standard deviation [SD] = stroke: $31-88 \mathrm{yr}, 64 \pm 16$ yr, TBI: $19-90 \mathrm{yr}$, $49 \pm 20.5$ yr) over 9 months at both facilities. Each patient subject was allowed to be observed for a maximum of three therapy sessions. Observations occurred during the patient subject's regularly scheduled occupational or physical therapy sessions, which addressed motor impairments related to the patient's diagnosis (not evaluations or discharge planning). Initial contact with the patient subject was made through the patient's primary therapist (provider subject). After initial contact was made, the observer explained the study to the patient subject and obtained informed consent before the observation. Neither the patient subjects nor the provider subjects knew that number of reps was being counted. Provider and patient subjects were told that the observers were "recording what happened during a therapy session.”

\section{Categories}

Data were collected by the counting of reps of a particular activity according to the following categories: UL, LL, and mobility. The UL and LL categories were further divided into the following subcategories: (1) active exercise, (2) passive exercise, (3) sensory, and (4) functional activity. Mobility was divided into the following subcategories: gait, transfers, stairs, wheelchair mobility, and balance (see Table 1 for complete description and examples; also see Lang et al. [17]). If during an instructed task more than one type of activity was performed, then each was counted, such as might occur when a patient performs a balance task that incorporates a UL functional movement. In addition, a repetition was counted if a clear attempt was made at the task; the task did not have to be completed in its entirety. We also documented duration of therapy session, patient diagnosis, side affected, age, and sex (Table 2). Each patient subject's Functional Independence Measure (FIM) score [22] on admission was documented when available. However, the entire FIM was not completed in the majority of cases and therefore could not be used in any statistical analysis. Thus, scores on FIM items were used but mean values for the total FIM score could not be calculated for each group. Range and mean of the documented FIM locomotor scores are reported in Table 2 to provide an example of locomotor skills at the baseline. Provider subject information was collected and included age, sex, years of experience, years of neurological rehabilitation experience, and degree earned. Of the 107 session observations, 6 were conducted by providers in the <1-year group, 40 by the 1 to $<5$-year group, 20 by the 5 to $<10$-year group, 13 by the 10 to $<15$-year group, and 28 by the $>15$-year group.

\section{Data Reduction}

Sessions that contained no entries in a given category or subcategory were eliminated from that category or subcategory for analyses. For example, if no UL exercises in any subcategory (active, passive, sensory, or functional) were instructed within a therapy session, we assumed that UL function was not the focus of the session and, thus, should not be included in the statistical comparison. Thus, different $n$ values (observed sessions) are assigned for some subcategories (Tables 2-4). Additionally, if $<10$ percent of the observed sessions contained a particular subcategory of intervention, this subcategory was eliminated from the comparison between groups. This was the case for sensory, passive exercise, and wheelchair mobility. These data are reported in the results tables but were not 
JRRD, Volume 47, Number 9, 2010

Table 1.

Description of activity within each subcategory.

\begin{tabular}{ll}
\hline Subcategory & \multicolumn{1}{c}{ Description } \\
\hline Passive Exercise & $\begin{array}{l}\text { Any joint movement that is done by therapist or outside source without active participation of patient. } \\
\text { Examples include but are not limited to stretching of wrist and finger flexors, stretching of ankle muscles, } \\
\text { and passive shoulder range of motion. }\end{array}$
\end{tabular}

Active Exercise Any movement in which patient partially or completely engages in moving limb from resting position, through specific movement, and back to resting position. Examples include but are not limited to raising arm out in front and returning it to side, flexing and extending elbow, bending knee and hip up to chest and then straightening out leg, adducting and abducting leg, and flexing ankle.

Functional Activity Any movement that produces end result or contributes to productivity. Examples include but are not limited to reaching or grasping for target object and activities of daily living, such as dressing, bathing, tooth brushing, and cooking. Many of these tasks were broken down into subunits for counting purposes. (Although gait and stairs are considered purposeful activities in clinical setting, they were counted separately for purposes of this study.)

Sensory Any activity that involves providing patient stimulation for sensation or proprioception. Examples include but are not limited to vibratory stimulation to muscle.

Gait Ambulation with or without assistive device. Recorded in episodes and individual steps within each episode. If patient rested for $>20$ seconds, new episode was recorded.

Transfers Any movement by patient from one place to another or change in position. Examples include but are not limited to sit to stand, stand to sit, mat to chair, supine to sit, chair to toilet, and stand to kneel. Transfers were only counted if they were specifically instructed by therapist.

Balance $\quad$ Any task instructed by therapist to maintain balance. Examples include but are not limited to standing or sitting with and without assistance. One episode of balance was counted for each balance task presented.

Wheelchair Propulsion Any activity that requires patient to navigate wheelchair independently. Recorded in episodes and repetitions within that episode counted as number of arm movements needed to propel wheelchair. If patient rested for $>20$ seconds, new episode was recorded.

Stairs

Any activity that requires patient to navigate single step-up or flight of stairs. Recorded in episodes and individual stair steps within each episode. If patient rested for $>20$ seconds, new episode was recorded.

Table 2.

Demographics for stroke and traumatic brain injury (TBI) patients.

\begin{tabular}{lcc}
\hline \multicolumn{1}{c}{ Demographic } & Stroke & TBI \\
\hline Age, Mean \pm SD (years) & $63.00 \pm 19.38$ & $51.34 \pm 19.32$ \\
Duration Between Injury \& Session (days) & & \\
$\quad$ Range & $5-300$ & $10-86$ \\
$\quad$ Mean & 42 (3 outliers $>100$ days) & 40 \\
Side Affected (\%) & & \\
$\quad$ Right & 42 & 12 \\
Left & 50 & 16 \\
Bilateral & 4 & 38 \\
$\quad$ Not Specified in Medical Chart & - & 29 \\
Sex (\%) & & \\
$\quad$ Male & 54 & 66 \\
Female & 46 & 33 \\
FIM Locomotor Score & & \\
$\quad \%$ Reported & 50 & $1-5$ \\
Range & $1-7$ & 2.55 \\
$\quad$ Mean & 2.91 & \\
\hline FIM = Functional Independence Measure, SD $=$ standard deviation. & \\
\hline \hline
\end{tabular}

compared between groups because of the very low observed frequency. Though data were collected from providers with four different licenses, the distribution of categories did not allow for comparisons between licensure. This is because, in the investigated settings, occupational therapy tended to focus more on UL issues and physical therapy more on LL. Thus, we pooled all provider subjects into one group.

\section{Statistical Analysis}

Data were assessed for normality. We used two metrics to examine the amounts of practice. First, we compared the number of reps per session between groups in each category and subcategory. We also computed and compared the reps per minute by dividing the total reps by the therapy time for each session. This second metric controlled for differences in total therapy time across 
Table 3.

Upper limb: Average total task repetitions (Reps) performed each rehabilitation session and each minute by traumatic brain injury (TBI) and stroke patients.

\begin{tabular}{|c|c|c|c|c|c|c|}
\hline \multirow[b]{2}{*}{ Task } & \multicolumn{3}{|c|}{ TBI $(n=27)$} & \multicolumn{3}{|c|}{ Stroke $(n=28)$} \\
\hline & $\begin{array}{l}\text { Mean Reps/ } \\
\text { Session }\end{array}$ & SD & $95 \%$ CI & $\begin{array}{l}\text { Mean Reps/ } \\
\text { Session }\end{array}$ & SD & $95 \%$ CI \\
\hline Active Exercise & 26.44 & 46.40 & 8.09 to 44.80 & 17.50 & 26.38 & 7.27 to 27.73 \\
\hline Passive Exercise & 11.93 & 32.10 & 0 to 24.63 & 5.43 & 11.07 & 1.13 to 9.72 \\
\hline Functional & 22.33 & 33.92 & 8.91 to 35.75 & 14.50 & 28.93 & 3.28 to 25.71 \\
\hline Sensory & 0 & 0 & 0 & 3.21 & 9.52 & 0 to 6.90 \\
\hline \multirow[t]{2}{*}{ Total } & 60.85 & 52.47 & 40.10 to 81.61 & 40.64 & 32.14 & 28.18 to 53.10 \\
\hline & Mean Reps/Min & & & Mean Reps/Min & & \\
\hline Active Exercise & 1.29 & 2.55 & 0.28 to 2.29 & 0.52 & 0.90 & 0.17 to 0.87 \\
\hline Passive Exercise & 0.60 & 1.81 & 0 to 1.31 & 0.19 & 0.42 & 2.82 to 0.36 \\
\hline Functional & 1.00 & 1.44 & 0.42 to 1.57 & 0.43 & 0.95 & 5.98 to 0.80 \\
\hline Sensory & 0 & 0 & 0 & 6.87 & 0.22 & 0 to 0.15 \\
\hline Total $^{*}$ & 2.88 & 2.83 & 1.76 to 4.00 & 1.21 & 1.19 & 0.75 to 1.67 \\
\hline
\end{tabular}

Table 4.

Lower limb: Average total task repetitions (Reps) performed each rehabilitation session and each minute by traumatic brain injury (TBI) and stroke patients.

\begin{tabular}{|c|c|c|c|c|c|c|}
\hline \multirow[b]{2}{*}{ Task } & \multicolumn{3}{|c|}{ TBI $(n=28)$} & \multicolumn{3}{|c|}{ Stroke $(n=24)$} \\
\hline & $\begin{array}{l}\text { Mean Reps/ } \\
\text { Session }\end{array}$ & SD & $95 \%$ CI & $\begin{array}{c}\text { Mean Reps/ } \\
\text { Session }\end{array}$ & SD & $95 \% \mathrm{CI}$ \\
\hline Passive Exercise & 1.46 & 3.13 & 0.25 to 2.68 & 1.38 & 3.51 & 0 to 2.86 \\
\hline Functional & 0.14 & 0.52 & 0 to 0.35 & 0.38 & 1.28 & 0 to 0.92 \\
\hline Sensory & 0.21 & 0.83 & 0 to 0.54 & 0.38 & 1.47 & 0 to 1.00 \\
\hline & \multicolumn{3}{|l|}{ Mean Reps/Min } & Mean Reps/Min & & \\
\hline Active Exercise & 3.24 & 5.37 & 1.16 to 5.32 & 0.99 & 1.50 & 0.31 to 1.62 \\
\hline Passive Exercise & 5.49 & 0.12 & 8.65 to 0.10 & 4.87 & 0.14 & 0 to 0.11 \\
\hline Functional & 6.74 & 2.49 & 0 to 1.64 & 1.34 & 0.05 & 0 to 3.44 \\
\hline Sensory & 8.04 & 2.97 & 0 to 0.02 & 9.76 & 3.57 & 0 to 0.02 \\
\hline
\end{tabular}

patients and groups. For comparisons between the stroke and TBI groups, either parametric two-tailed $t$-tests or Mann-Whitney $\mathrm{U}$ independent two-tailed $t$-tests were conducted, as appropriate. Because of nonnormal distribution, Kruskal-Wallis one-way analysis of variance was applied to compare therapist years of experience and number of reps. For all data, an alpha level of 0.05 was used and results are presented as mean \pm SD unless otherwise indicated. Lastly, correlation and regression analyses were used to determine whether any relationships existed between the number of reps performed and specific patient demographics and characteristics (e.g., age, FIM UL item scores, FIM locomotor score, time since onset) in each of the categories and subcategories. 


\section{RESULTS}

\section{Overview of Data from Both Groups}

Pooling the data from both groups, the average total number of UL reps across all four subcategories (active exercise, passive exercise, sensory, and functional activity) in those sessions that included UL rehabilitation ( $n=$ 55) was $50.56 \pm 44.11$. The average total number of reps for each of the subcategories was $18.34 \pm 31.43$ for UL functional activity, $21.89 \pm 37.48$ for UL active exercise, $8.62 \pm 23.84$ for UL passive exercise, and $1.64 \pm 6.92$ for UL sensory. With both groups pooled, the average total number of LL reps across all four subcategories in those sessions that included LL rehabilitation $(n=52)$ was $61.04 \pm 102.75$. The average total number of reps for the subcategories was $0.25 \pm 0.95$ for LL functional activity, $59.08 \pm 103.58$ for LL active exercise, $1.42 \pm 3.28$ for LL passive exercise, and $0.29 \pm 1.16$ for LE sensory. The average number of gait reps in those sessions that included gait $(n=58)$ was $249.28 \pm 254.47$. Therapy sessions lasted on average $29.11 \pm 12.14$ minutes.

\section{Comparison between TBI and Stroke Groups}

\section{Upper and Lower Limbs}

Tables 3 and $\mathbf{4}$ provide the number of reps per session and the number of reps per minute for each UL and LL category and subcategory. No differences in reps per session were found between groups ( $p$-values $>0.05$ ) for any of the UL and LL categories and subcategories. In comparisons of reps per minute, the TBI group had more total UL reps per minute than the stroke group $(p<0.05)$. No other differences in reps per minute were found.

\section{Mobility}

Numbers of reps per session and per minute for the mobility categories are provided in Table 5. When examining the mobility categories with the reps per session metric, we found no differences between groups ( $p$-values $>0.05$ ). When examining the mobility categories with the reps per minute metric, we found that reps per minute were higher in the TBI group than the stroke group for the gait steps and transfers categories ( $p$-values $<0.05$ ).

\section{Patient Characteristics}

To examine the effects of patient age and functional status on the number of reps per session, correlation and regression analyses were performed for each category. Correlation coefficients ranged from -0.0019 to 0.0256 ( $p$-values $>0.05)$. The regression model indicated that patient factors predicted little variance in the number of reps performed $\left(R^{2}=0.06, p>0.05\right)$.

\section{Therapist Experience}

A statistically significant difference was found for years of neurorehabilitation experience of therapists for two

Table 5.

Mobility: Average total task repetitions (Reps) performed each rehabilitation session and each minute by traumatic brain injury (TBI) and stroke patients.

\begin{tabular}{|c|c|c|c|c|c|c|c|c|}
\hline \multirow[b]{2}{*}{ Task } & \multicolumn{4}{|c|}{ TBI } & \multicolumn{4}{|c|}{ Stroke } \\
\hline & $n$ & $\begin{array}{c}\text { Mean Reps/ } \\
\text { Session }\end{array}$ & SD & $95 \%$ CI & $n$ & $\begin{array}{c}\text { Mean Reps/ } \\
\text { Session }\end{array}$ & SD & $95 \%$ CI \\
\hline Gait Steps & 28 & 317.93 & 330.26 & 180.86 to 445.99 & 30 & 185.20 & 130.1 & 136.63 to 233.77 \\
\hline Transfers & 31 & 9.32 & 15.02 & 3.81 to 14.83 & 43 & 7.81 & 6.10 & 594.00 to 9.70 \\
\hline Balance & 23 & 46.70 & 64.62 & 18.75 to 74.64 & 27 & 23.07 & 25.91 & 12.82 to 33.32 \\
\hline Stairs & 4 & 28.75 & 22.08 & 0 & 9 & 37.60 & 33.04 & 13.97 to 61.23 \\
\hline & & Mean Reps/Mir & & & & Mean Reps/Mi & & \\
\hline Gait Steps ${ }^{*}$ & 28 & 13.13 & 14.08 & 7.66 to 18.59 & 30 & 4.97 & 3.50 & 3.67 to 6.28 \\
\hline Transfers ${ }^{*}$ & 31 & 0.39 & 0.54 & 0.19 to 0.59 & 43 & 0.22 & 0.14 & 0.18 to 0.26 \\
\hline Balance & 23 & 1.88 & 2.37 & 0.86 to 2.91 & 27 & 0.67 & 0.85 & 0.35 to 1.03 \\
\hline
\end{tabular}


comparisons $(p=0.02)$. Therapists in the 5 to $<10$-year and 10 to $<15$-year groups instructed patients in significantly more functional reps per minute than did therapists in the $<1$-year group $(z=1.96$ and $z=3.00)$. Therapists in the 10 to $<15$-year group also instructed patients in significantly more functional reps per minute than did therapists in the $>15$-year group $(z=2.22)$ (Figure). No differences were found in the other categories and subcategories assessed.

\section{DISCUSSION}

Results of this study demonstrate that in the observed rehabilitation sessions, the number of reps performed per session (e.g., between 40-60 reps for all UL categories) did not approach that which neuroplasticity research has suggested is required for cortical reorganization. Though no specific amount has been established, the evidence across animal and human literature suggests that the number is in the hundreds for the UL [23] and in the thousands for gait steps [10]. Ours is the first report on this issue in patients with TBI, and our finding is consistent with results found in a multicenter study in subjects with stroke [17].

We also evaluated reps per minute to account for any differences in total treatment time and for differences in

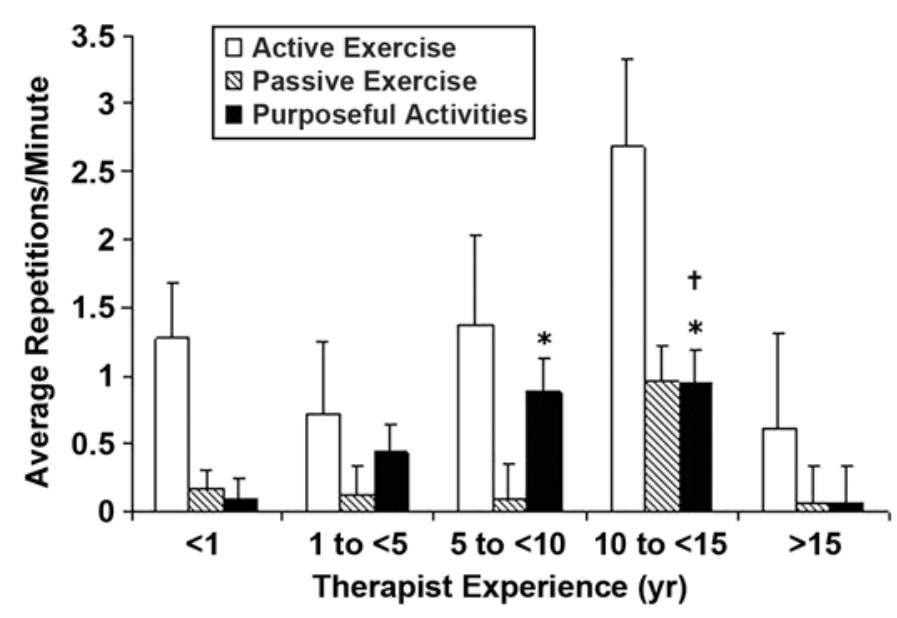

Figure.

Instructed repetitions by therapists according to years of neurological rehabilitation experience. Therapists with 5 to $<10$ years and 10 to $<15$ years of experience instructed patients in significantly more purposeful activities than therapists with $<1$ year of experience $\left({ }^{*} p<\right.$ 0.05 ). Therapists with 10 to $<15$ years of experience instructed patients in significantly more purposeful activities than therapists with $>15$ years of experience $\left({ }^{\dagger} p<0.05\right)$. how a therapist may choose to distribute the activities performed in a given therapy session. When evaluated in this manner, the TBI group performed more total UL reps per minute, gait steps per minute, and transfers per minute than the stroke group. The variability in reps was quite high within groups, which may have accounted for different conclusions from the reps per session versus reps per minute data. Since observation often influences behavior, the number of reps counted here may overestimate "typical" rehabilitation if provider and patient subjects were hoping to favorably impress the observer.

Is repetition a valid measure of practice? Though not fully understood, the number of reps appears to be an important issue for functional improvement and cortical reorganization [24]. We posit that comparison of reps across types of injury in the same species (humans), such as was done here, is reasonable. While the direct translation of exact numbers across species may not be valid, the translation of general estimates probably is valid. Our rationale is that (1) the relative contributions of various motor system structures, such as the rubrospinal tract [25], differ in humans compared with monkeys and rats (see also Nudo and Masterton for data [26] and Lang et al. for review [27]) and (2) monkey and rat models are not exact replications of the human experience of stroke and TBI. Thus, one could conclude that while the animal models indicate that large numbers of reps may be required, they do not specify what exactly those numbers need to be.

Other issues are also likely to be important, such as the effort required to perform a task and how meaningful the task is to the patient. This study did not attempt to answer these less measurable issues but did attempt to control for the issue of severity of motor impairment by counting a partial performance of a movement as a repetition. One interesting finding in our results and in the multisite study with stroke patients [17] was that motor function, as measured by FIM scores, was not related to reps. Thus, the clinical perception that people who are more severely affected do fewer reps was not supported. This finding may mean that the observed therapists were skilled at grading the activities to the capabilities of each patient. In a study specifically addressing the issue of simple repetitive movement versus repetitive movement requiring more active cognitive processing, two groups with stroke performed a finger-tracking task that was either an "easy" highly repetitive task or a "difficult" repetitive task that required visuospatial processing and motor learning [12]. Contrary 
to the authors' hypothesis, both groups improved on the functional tests. In fact, the group that performed the easy task had greater improvement on one of the functional tests. The authors speculated that the lack of advantage of the more difficult training was due to the "higher number of reps" performed by the group completing the easy task. Both groups trained for the same amount of time and at the same range of motion, but the group with the easy training was able to self-pace and performed at a statistically higher frequency of movement and, thus, performed a higher number of reps than the group with the difficult motorlearning task [12].

In the current study, no difference was found between groups when they were evaluated with the reps per session metric. This metric of amount is the most appropriate for comparison to the neuroplasticity research previously discussed. We did find, however, several differences between groups when they were evaluated with the reps per minute metric. While the design of the study did not allow specific investigation of the reasons for these differences between groups, several factors may have contributed, such as cognitive, behavioral, or age-related issues. Although no correlation was found between patient age and number of reps performed, generally, patients with TBI tend to be younger males and patients with stroke tend to be older. Patients with stroke are more likely to have comorbidities, such as concurrent vascular disease, that may affect their ability to perform the same number of reps or to perform at the same pace as TBI patients without vascular disease. Alternatively, therapists may be less likely to challenge older patients to the same degree as younger patients with similar levels of impairment.

Years of experience in the area of neurological rehabilitation significantly affected the amount of practice received. Providers with 5 to $<15$ years of experience instructed their patients in significantly more functional activities than did those with $<1$ year of experience. Providers with 10 to $<15$ years of experience instructed patients in significantly more functional reps than did providers with $>15$ years of experience. The differences in reps between providers suggest that those with less experience may have less confidence in how to best facilitate neuroplastic change through functional motor tasks or may lack the experience or "bag of tricks" needed to creatively facilitate the task in a functional way. With less experience, one may feel less comfortable challenging a patient to perform more reps or perform a more complex or cognitively challenging motor task. A new therapist may also have difficulty modifying traditional motor tasks to make them more functional yet still achievable by patients with limited abilities. Perhaps the more experienced therapists were able to devise exercises that addressed two components, such as a balance activity with a functional UL movement, which would increase the number of reps a given patient performed. Therapists with $>15$ years of experience in neurological rehabilitation may have been educated under a different model in which use of functional tasks during therapy was not emphasized.

The question in many clinicians' minds may be, Is it feasible to perform a high number of reps? Evidence that this can be successfully employed exists in CIMT literature (for a review, see Wolf et al. [28]) and in 1-hour therapy sessions in a recent cohort of people with chronic stroke [29]. Specific to TBI, Canning et al. documented the effects of additional therapeutic reps on functional performance [21]. The researchers required the experimental group to perform an average of 87 additional sit-to-stand reps and 42 additional step-up exercises per day compared with the standard-care control group. A 62 percent improvement in motor performance was reported in all 12 subjects in the experimental group compared with an 18 percent improvement in the control group $(p=0.05)$. These results demonstrate that increasing the number of reps during therapy sessions results in desirable outcomes. When specific treatment goals are used within each session, a much higher number of reps per session can be accomplished [29]. Indeed, other tools or models of therapy may need to be considered rather than the traditional one-on-one interaction. Group or robot-assisted therapies are areas currently under investigation that may support the goal of increased numbers of reps (for a review, see Kwakkel et al. [30]). Activities emphasizing changes to both the neuroplastic mechanism and the muscle must be considered for optimal effectiveness. Historically, strength training and rangeof-motion exercises have been the focus of rehabilitation. For a deconditioned patient, increasing strength is an important goal; however, it has been shown in subjects with stroke that a significant increase in strength does not necessarily result in improved functional performance [31] or cortical change as measured by functional magnetic resonance imaging [32]. Physical and occupational therapists may need to reexamine the goal of therapy sessions to determine how to best facilitate muscle strengthening with motor learning, planning, and control.

Given the preliminary and observational nature of this study, results cannot be necessarily interpreted as representing the population at large. However, in the preliminary 
work by Lang et al., the results of a single site of observation [16] were supported by the larger international study [17], indicating that consistencies exist in rehabilitation practice regardless of region or setting. Another study limitation includes the lack of information regarding the functional status of each patient subject. Other than FIM score, which has been shown to be an insensitive predictor [33], functional status information is not typically collected during rehabilitation sessions and, thus, reflects a limitation of our rehabilitation system. In addition, this study documented the types and number of reps performed but did not address many other issues that may affect functional improvement, such as "quality" versus "quantity" of movement, duration, types of feedback or cues given, and the cognitive demand of the task. Nevertheless, our study presents a starting point for a more in-depth investigation into other critical issues, such as ideal therapy dose.

\section{CONCLUSIONS}

This study shows that in the rehabilitation of patients after TBI and stroke, (1) considerably fewer total reps are performed in any category compared with what neuroplasticity research suggests is required for neuroplastic change, (2) slight differences in reps per minute occur based on patient diagnosis, and (3) emphasis on reps of functional activity varies based on therapist experience. These findings are important for researchers in the field of neuroplasticity to consider in the general framework from which therapy is being provided and within the confines of the current clinical setting. In addition, rehabilitation professionals must examine other models of service delivery to find creative solutions for achieving more practice. These models may include group therapy, circuit training [34], or alterations in daily therapy schedules to allow longer sessions each day. If sessions are organized so as to maximize reps, the patient may be more likely to rebuild necessary cortical pathways through neuroplasticity and achieve greater functional improvement.

\section{ACKNOWLEDGMENTS}

\section{Author Contributions:}

Study concept and design: T. J. Kimberley, C. E. Lang. Acquisition of data: S. Samargia, L. G. Moore, J. K. Shakya. Analysis and interpretation of data: T. J. Kimberley, S. Samargia, L. G. Moore, J. K. Shakya, C. E. Lang.
Drafting of manuscript: T. J. Kimberley.

Critical revision of manuscript for important intellectual content:

T. J. Kimberley, S. Samargia, L. G. Moore, J. K. Shakya, C. E. Lang. Statistical analysis: S. Samargia, L. G. Moore, J. K. Shakya,

T. J. Kimberley.

Study supervision: T. J. Kimberley.

Financial Disclosures: The authors have declared that no competing interests exist. Parts of this work were presented at the Combined Sections Meeting of the American Physical Therapy Association, February 2009, Las Vegas, Nevada.

Funding/Support: This material was unfunded at the time of manuscript preparation. Salary support for Dr. Lang was provided by the National Institutes of Health (grant HD047669).

Additional Contributions: We would like to thank Amy Kieffer, PT; Carol McKenzie, OT; and Tricia Nichols, MS, PT, for their valuable assistance.

Institutional Review: This study was approved by the University of Minnesota Internal Review Board and the Health East Internal

Review Board. All subjects gave informed written consent according to the Declaration of Helsinki.

Participant Follow-Up: The authors do not plan to inform participants of the publication of this study because contact information is unavailable.

\section{REFERENCES}

1. Nudo RJ. Adaptive plasticity in motor cortex: Implications for rehabilitation after brain injury. J Rehabil Med. 2003; (41 Suppl):7-10. [PMID: 12817650$]$ DOI:10.1080/16501960310010070

2. Kleim JA, Barbay S, Nudo RJ. Functional reorganization of the rat motor cortex following motor skill learning. J Neurophysiol. 1998;80(6):3321-25. [PMID: 9862925]

3. Nudo RJ, Milliken GW, Jenkins WM, Merzenich MM. Use-dependent alterations of movement representations in primary motor cortex of adult squirrel monkeys. J Neurosci. 1996;16(2):785-807. [PMID: 8551360$]$

4. Plautz EJ, Milliken GW, Nudo RJ. Effects of repetitive motor training on movement representations in adult squirrel monkeys: Role of use versus learning. Neurobiol Learn Mem. 2000;74(1):27-55. [PMID: 10873519] DOI:10.1006/nlme.1999.3934

5. Classen J, Liepert J, Wise SP, Hallett M, Cohen LG. Rapid plasticity of human cortical movement representation induced by practice. J Neurophysiol. 1998;79(2):1117-23. [PMID: 9463469]

6. Carey JR, Kimberley TJ, Lewis SM, Auerbach EJ, Dorsey L, Rundquist P, Ugurbil K. Analysis of fMRI and finger tracking training in subjects with chronic stroke. Brain. 2002;125(Pt 4):773-88. [PMID: 11912111]

DOI:10.1093/brain/awf091

7. Liepert J, Graef S, Uhde I, Leidner O, Weiller C. Traininginduced changes of motor cortex representations in stroke 
patients. Acto Neurol Scand. 2000;101(5):321-26.

[PMID: 10987321]

DOI:10.1034/j.1600-0404.2000.90337a.X

8. Liepert J, Miltner WH, Bauder H, Sommer M, Dettmers C, Taub E, Weiller C. Motor cortex plasticity during constraintinduced movement therapy in stroke patients. Neurosci Lett. 1998;250(1):5-8. [PMID: 9696052]

DOI:10.1016/S0304-3940(98)00386-3

9. Cha J, Heng C, Reinkensmeyer DJ, Roy RR, Edgerton VR, De Leon RD. Locomotor ability in spinal rats is dependent on the amount of activity imposed on the hindlimbs during treadmill training. J Neurotrauma. 2007;24(6):1000-12. [PMID: 17600516] DOI:10.1089/neu.2006.0233

10. Chau C, Barbeau H, Rossignol S. Early locomotor training with clonidine in spinal cats. J Neurophysiol. 1998;79(1): 392-409. [PMID: 9425208]

11. De Leon RD, Hodgson JA, Roy RR, Edgerton VR. Locomotor capacity attributable to step training versus spontaneous recovery after spinalization in adult cats. J Neurophysiol. 1998;79(3):1329-40. [PMID: 9497414]

12. Carey JR, Durfee WK, Bhatt E, Nagpal A, Weinstein SA, Anderson KM, Lewis SM. Comparison of finger tracking versus simple movement training via telerehabilitation to alter hand function and cortical reorganization after stroke. Neurorehabil Neural Repair. 2007;21(3):216-32.

[PMID: 17351083]

DOI:10.1177/1545968306292381

13. Wolf SL, Winstein CJ, Miller JP, Taub E, Uswatte G, Morris D, Giuliani C, Light KE, Nichols-Larsen D, EXCITE Investigators. Effect of constraint-induced movement therapy on upper extremity function 3 to 9 months after stroke: The EXCITE randomized clinical trial. JAMA. 2006; 296(17):2095-2104. [PMID: 17077374$]$

14. English CK, Hillier SL, Stiller KR, Warden-Flood A. Circuit class therapy versus individual physiotherapy sessions during inpatient stroke rehabilitation: A controlled trial. Arch Phys Med Rehabil. 2007;88(8):955-63. [PMID: 17678655] DOI:10.1016/j.apmr.2007.04.010

15. Kuys S, Brauer S, Ada L. Routine physiotherapy does not induce a cardiorespiratory training effect post-stroke, regardless of walking ability. Physiother Res Int. 2006; 11(4):219-27. [PMID: 17236529]

DOI:10.1002/pri.344

16. Lang CE, MacDonald JR, Gnip C. Counting repetitions: An observational study of outpatient day treatment for people with hemiparesis post-stroke. J Neurol Phys Ther. 2007; 31(1):3-10. [PMID: 17419883]

17. Lang CE, Macdonald JR, Reisman DS, Boyd L, Jacobson Kimberley T, Schindler-Ivens SM, Hornby TG, Ross SA, Scheets PL. Observation of amounts of movement practice provided during stroke rehabilitation. Arch Phys Med
Rehabil. 2009;90(10):1692-98. [PMID: 19801058]

DOI:10.1016/j.apmr.2009.04.005

18. Rutland-Brown W, Langlois JA, Thomas KE, Xi YL. Incidence of traumatic brain injury in the United States, 2003. J Head Trauma Rehabil. 2006;21:544-48. [PMID: 17122685] DOI:10.1097/00001199-200611000-00009

19. Hoge CW, McGurk D, Thomas JL, Cox AL, Engel CC, Castro CA. Mild traumatic brain injury in U.S. Soldiers returning from Iraq. N Engl J Med. 2008;358(5):453-63. [PMID: 18234750] DOI:10.1056/NEJMoa072972

20. Shaw SE, Morris DM, Uswatte G, McKay S, Meythaler JM, Taub E. Constraint-induced movement therapy for recovery of upper-limb function following traumatic brain injury. J Rehabil Res Dev. 2005;42(6):769-78.

[PMID: 16680614] DOI:10.1682/JRRD.2005.06.0094

21. Canning CG, Shepherd RB, Carr JH, Alison JA, Wade L, White A. A randomized controlled trial of the effects of intensive sit-to-stand training after recent traumatic brain injury on sit-to-stand performance. Clin Rehabil. 2003; 17(4):355-62. [PMID: 12785242$]$ DOI:10.1191/0269215503cr620oa

22. Keith RA, Granger CV, Hamilton BB, Sherwin FS. The Functional Independence Measure: A new tool for rehabilitation. Adv Clin Rehabil. 1987;1:6-18. [PMID: 3503663]

23. Nudo RJ, Wise BM, SiFuentes F, Milliken GW. Neural substrates for the effects of rehabilitative training on motor recovery after ischemic infarct. Science. 1996;272(5269): 1791-94. [PMID: 8650578] DOI:10.1126/science.272.5269.1791

24. Bütefisch C, Hummelsheim H, Denzler P, Mauritz KH. Repetitive training of isolated movements improves the outcome of motor rehabilitation of the centrally paretic hand. J Neurol Sci. 1995;130(1):59-68. [PMID: 7650532] DOI:10.1016/0022-510X(95)00003-K

25. Nathan PW, Smith MC. The rubrospinal and central tegmental tracts in man. Brain. 1982;105(Pt 2):223-69. [PMID: 7082990] DOI:10.1093/brain/105.2.223

26. Nudo RJ, Masterton RB. Descending pathways to the spinal cord: A comparative study of 22 mammals. J Comp Neurol. 1988;277(1):53-79. [PMID: 3198796] DOI:10.1002/cne.902770105

27. Lang CE, Reilly KT, Schieber MH. Human voluntary motor control and dysfunction. In: Selzer M, Clarke S, Cohen L, Duncan P, Gage F, editors. Textbook of neural repair and rehabilitation. Vol II. Cambridge (UK): Cambridge University Press; 2006.

28. Wolf SL, Blanton S, Baer H, Breshears J, Butler AJ. Repetitive task practice: A critical review of constraint-induced movement therapy in stroke. Neurologist. 2002;8(6):325-38. 
[PMID: 12801434$]$

DOI:10.1097/00127893-200211000-00001

29. Birkenmeier RL, Prager EM, Lang CE. Translating animal doses of task-specific training to people with chronic stroke in 1-hour therapy sessions: A proof-of-concept study. Neurorehabil Neural Repair. 2010;24(7):620-35 [PMID: 20424192]

30. Kwakkel G, Kollen BJ, Krebs HI. Effects of robot-assisted therapy on upper limb recovery after stroke: A systematic review. Neurorehabil Neural Repair. 2008;22(2):111-21. [PMID: 17876068$]$ DOI:10.1177/1545968307305457

31. Van Peppen RP, Kwakkel G, Wood-Dauphinee S, Hendriks HJ, Van der Wees PJ, Dekker J. The impact of physical therapy on functional outcomes after stroke: What's the evidence? Clin Rehabil. 2004;18(8):833-62. [PMID: 15609840] DOI:10.1191/0269215504cr843oa

32. Kimberley TJ, Lewis SM, Auerbach EJ, Dorsey LL, Lojovich JM, Carey JR. Electrical stimulation driving functional improvements and cortical changes in subjects with stroke. Exp Brain Res. 2004;154(4):450-60. [PMID: 14618287] DOI:10.1007/s00221-003-1695-y

33. Lin KC, Huang YH, Hsieh YW, Wu CY. Potential predictors of motor and functional outcomes after distributed con- straint-induced therapy for patients with stroke. Neurorehabil Neural Repair. 2009;23(4):336-42. [PMID: 18984830]

DOI:10.1177/1545968308321773

34. Elson T, English C, Hillier S. How much physical activity do persons recovering from stroke do during physiotherapy sessions? Int J Ther Rehabil. 2009;16(2):78-84.

Submitted for publication February 25, 2010. Accepted in revised form July 6, 2010.

This article and any supplementary material should be cited as follows:

Kimberley TJ, Samargia S, Moore LG, Shakya JF, Lang CE. Comparison of amounts and types of practice during rehabilitation for traumatic brain injury and stroke. J Rehabil Res Dev. 2010;47(9):851-62.

DOI:10.1682/JRRD.2010.02.0019

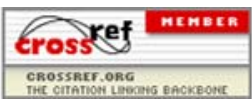


